\title{
On the Front (Phone) Lines: Results of a COVID-19 Hotline
}

\author{
David Margolius, MD, Mary Hennekes, BS, Jimmy Yao, BA, \\ Douglas Einstadter, MD, MPH, Douglas Gunzler, PhD, Nabil Chehade, MD, \\ Ashwini R. Sehgal, MD, Yasir Tarabichi, MD, MSCR, and Adam T. Perzynski, PhD
}

Background: Severe acute respiratory syndrome coronavirus (SARS-CoV-2) and the associated coronavirus disease of 2019 (COVID-19) have presented immense challenges for health care systems. Many regions have struggled to adapt to disruptions to health care practice and use systems that effectively manage the demand for services.

Methods: This was a cohort study using electronic health records at a health care system in northeast Ohio that examined the effectiveness of the first 5 weeks of a 24/7 physician-staffed CoVID-19 hotline including social care referrals for patients required to self-isolate. We describe clinical diagnosis, patient characteristics (age, sex race/ethnicity, smoking status, insurance status), and visit disposition. We use logistic regression to evaluate associations between patient characteristics, visit disposition and subsequent emergency department use, hospitalization, and SARS-Cov-2 PCR testing.

Participants: In 5 weeks, 10,112 patients called the hotline (callers). Of these, 4213 (42\%) were referred for a physician telehealth visit (telehealth patients). Mean age of callers was 42 years; $67 \%$ were female, $51 \%$ white, and $46 \%$ were on Medicaid/uninsured.

Results: Common caller concerns included cough, fever, and shortness of breath. Most telehealth patients (79\%) were advised to self-isolate at home, $14 \%$ were determined to be unlikely to have COVID-19, $3 \%$ were advised to seek emergency care, and $4 \%$ had miscellaneous other dispositions. A total of 287 patients (7\%) had a subsequent emergency department visit, and $44(1 \%)$ were hospitalized with a COVID-19 diagnosis. Of the callers, $482(5 \%)$ had a COVID-19 test reported, with 69 (14\%) testing positive. Among patients advised to stay at home, $83 \%$ had no further face-to-face visits. In multivariable results, only a physician recommendation to seek emergency care was associated with emergency department use (odds ratio $=4.73,95 \%$ confidence interval $=$ 1.37-16.39, $P=.014)$. Only older age was associated with having a positive test result. Patients with social needs and interest in receiving help were offered services to meet their needs including food deliveries $(n=92)$, behavioral health telephone visits $(n=49)$, and faith-based comfort calls from pastoral care personnel $(n=37)$.

Conclusions and Relevance: Robust, physician-directed telehealth services can meet a wide range of clinical and social needs during the acute phase of a pandemic, conserving scarce resources such as personal protective equipment and testing supplies and preventing the spread of infections to patients and health care workers. (J Am Board Fam Med 2021;34:S95-S102.)

Keywords: Cohort Studies, COVID-19, Health Care Systems, Hotlines, Logistic Models, Ohio, Pandemics, Telemedicine

\section{Introduction}

The coronavirus disease of 2019 (COVID-19) is caused by a novel pathogen (SARS-CoV-2) first

This article was externally peer reviewed.

Submitted 18 May 2020; revised 13 August 2020; accepted 13 August 2020.

From The MetroHealth System, Population Health Research Institute; and Case Western Reserve University, School of Medicine.

Funding: The MetroHealth System.

Disclosures: Dr Perzynski reports equity ownership of Global Health Metrics, LLC, and book royalties from Springer Nature and Taylor Francis all unrelated to and outside the work of this manuscript. Dr Gunzler reports detected in Wuhan, China, in December 2019. ${ }^{1}$ This pathogen has spread worldwide with $>3$ million infected and $>900,000$ cases and $>50,000$ deaths in the United States ${ }^{2}$ before the end of April

book royalties from Taylor Francis unrelated to and outside the work of this manuscript. The other authors have no conflicts to disclose.

Corresponding author: Adam T. Perzynski, PhD, Associate Professor of Medicine and Sociology, Center for Health Care Research and Policy, The MetroHealth System, Case Western Reserve University, 2500 MetroHealth Drive, R225A, Cleveland, OH 44109-1998 (E-mail: Adam. Perzynski@case.edu). 
2020. Although only a minority of patients with COVID-19 develop severe disease, ${ }^{3}$ researchers and clinicians have struggled to adapt to the cascade of disruptions to health care practice and to use systems that effectively manage the demand for services. Local, national, and international policy makers and health system leaders around the globe are urgently in need of evidence to guide decision making on effective clinical care strategies as a component of pandemic response.

During the $2009 \mathrm{H} 1 \mathrm{~N} 1$ pandemic, nurse triage lines reduced in-person visits for influenza-like symptoms, were cost effective, had a high degree of satisfaction among callers, and were able to reach rural and uninsured populations. ${ }^{4-7}$ Reaching vulnerable populations and those of lower socioeconomic status during a pandemic is of particular importance because individuals of lower socioeconomic status have greater barriers to information access and are more likely to adopt incorrect protective behaviors. ${ }^{8}$ The extent to which hotline services during the COVID-19 pandemic have been able to reach and provide effective care to uninsured populations has not been previously investigated. Given the disproportionate burden of COVID-19 in low-income and racial and ethnic minority communities, it is important to examine the extent to which these populations are reached by telehealth services.

To further promote telehealth in the COVID19 era, throughout March and April 2020, federal and state policies have been altered to remove or ease geographic restrictions and other regulatory barriers to telehealth visits. ${ }^{9}$ Telehealth is well suited for use during pandemics because clinicians can provide care and consultation to isolated populations and counter the surge in demand for medical care while using telehealth as a form of electronic personal protective equipment. ${ }^{9-13}$ Telehealth in the COVID-19 era is thus theorized as a useful strategy in forward triage, the sorting of patients before their arrival in the emergency department ${ }^{13,14}$ and as a means to provide patient guidance and reassurance. ${ }^{11}$

On Monday, March 9, the first 3 cases of COVID-19 were diagnosed in northeast Ohio. ${ }^{15}$ On March 13, our health care system launched a registered nurse (RN) triage-linked, 24-hour availability, physician-staffed hotline to assess, advise, and treat individuals who called with symptoms that could be COVID-19 related. Over the next 5 weeks, the $\mathrm{RN}$ triage line received more than 12,000 calls, resulting in more than 5000 physician telehealth visits. Whereas other institutions have reported on the establishment of triage system protocols within their electronic health records and have updated patient portals to provide patients with self-triage and self-scheduling abilities, ${ }^{15-18}$ to our knowledge this is the first study to examine use of a COVID-19 hotline that provides patients with direct physician care. We examined the extent to which a physician-staffed hotline succeeded in providing services across a diverse population and sought to understand the effectiveness of this form of telehealth in managing patients concerned about symptoms and exposure. Our analyses examined which patient characteristics and clinical care recommendations were associated with subsequent use of clinical services (emergency department, hospitalization, SARS-CoV-2 testing) and with SARS$\mathrm{CoV}-2$ test positivity.

\section{Methods}

\section{Overview}

This is a cohort study of patients who called a COVID-19 hotline at MetroHealth, a large urban safety-net health care system in Cuyahoga County in northeast Ohio. The study was approved by the MetroHealth Institutional Review Board.

\section{Setting}

This study took place at a publicly owned academic health care system in Cleveland, $\mathrm{OH}$, and surrounding municipalities that disproportionately serves a low-income, Medicaid, and uninsured population. The system employs more than 7800 individuals and consists of 4 hospitals, 4 emergency departments, and 20 health centers. In 2019, the system delivered care to more than 298,000 unique patients. According to the Ohio Department of Health, Cuyahoga County has among the highest number of COVID-19 cases among Ohio counties and the highest number of deaths. Cuyahoga County (including the city of Cleveland) is also among the most densely populated and socioeconomically disadvantaged communities in the United States. $^{19}$

\section{Intervention}

All individuals in northeast Ohio with questions or concerns about COVID-19 were invited to call a 
dedicated hotline if they had questions or concerns about COVID-19 (Figure 1). Callers are prompted to press 1 to hear information about COVID-19 or press 2 to speak with a nurse about questions or signs and symptoms of COVID-19. Interpreter services from a contracted telephonic interpreter service were available for all patients. Using standard protocols based on the caller's symptoms, the nurse determined whether further evaluation for COVID-19 was warranted and, if so, scheduled the patient for a same-day telehealth visit with a physician. A total of 91 physicians from family medicine, medicine-pediatrics, general internal medicine, pediatrics, and otolaryngology have staffed the hotline. The costs of this effort were all attributed to labor from 2 disciplines: the nurses answering calls and the physicians taking the telephone visits. Nurses answered calls as part of their regular work shifts providing telephone support to patients across the care system. Physicians signed up for shifts outside their regular clinic hours and were paid approximately $\$ 40$ per hour. Visits were billed as telehealth visits using 99441 (6-10 minutes), 99442 (11-20 minutes), and 99443 (21+ minutes) billing codes based on time spent on the phone.

Physicians evaluated the patient's condition over the phone and recommended a treatment plan. Patients who completed a physician telehealth visit received a follow-up call from a care coordinator within 24 hours to assess for any change in symptoms. During the follow-up call, the care coordinator also assessed the patient for limitations in basic living needs and offered to connect them with services provided by the MetroHealth Institute for H.O.P.E. (Health, Opportunities, Partnerships, Empowerment), including home delivery of food and prescriptions, behavioral health visits, and spiritual care.

\section{Measures}

We queried the electronic health record (Epic; Verona, WI) to extract data on patients who called the COVID-19 hotline and completed nurse triage only or were referred for a telehealth visit with a physician. We collected patient characteristics (age, sex, race/ethnicity, insurance type, smoking status) and clinical variables directly relevant to understanding the social epidemiology of the COVID-19 hotline

Figure 1. CoVID-19 Hotline Patient Flow.

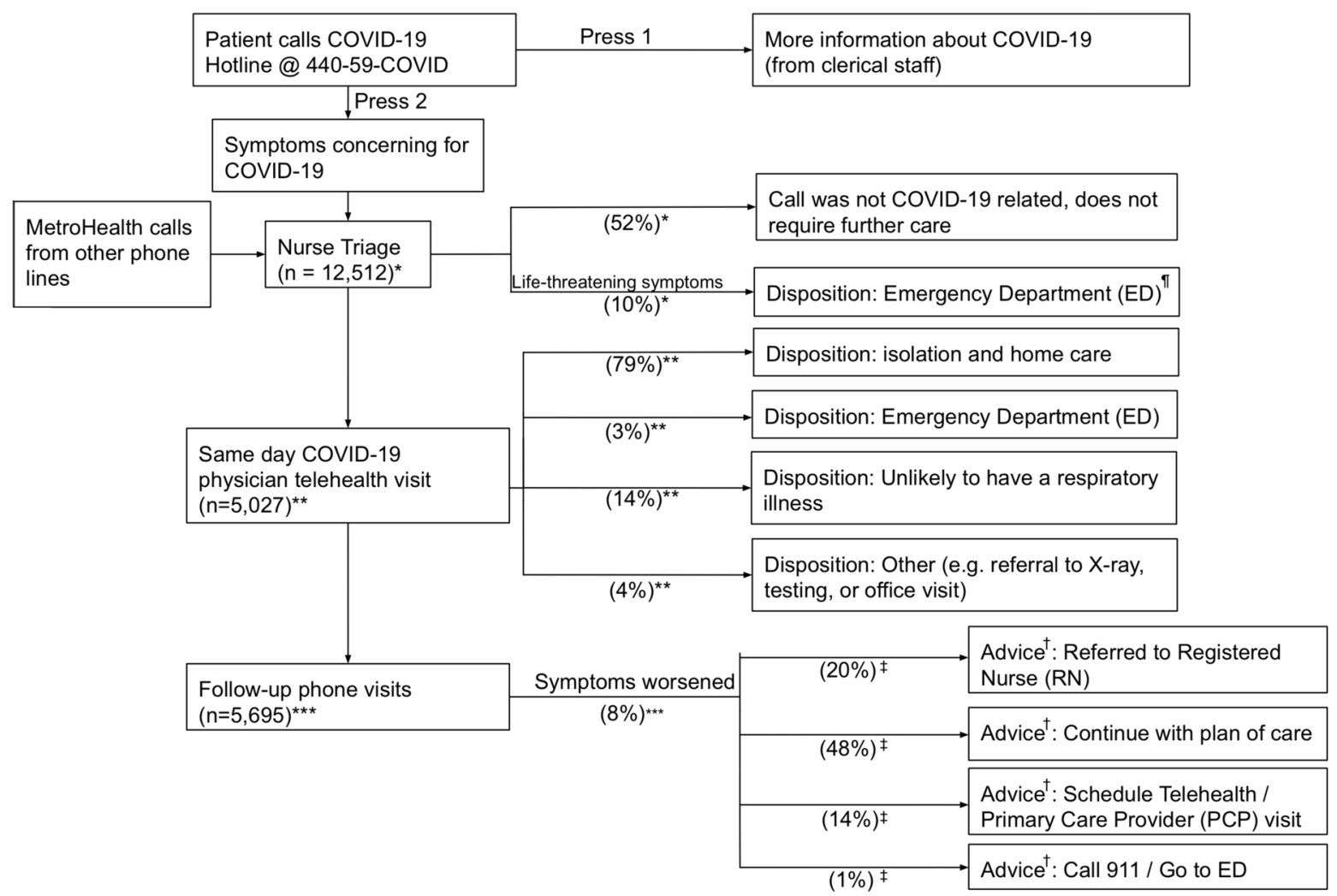


(symptom protocols, visit disposition, visit diagnoses). We also analyzed data from follow-up calls with regard to changes in health status and self-described basic needs. Race and ethnicity categories included non-Hispanic white, non-Hispanic black, Hispanic, and other. Insurance type categories included Medicaid, Medicare, commercial, employee insurance (a form of commercial insurance covering The MetroHealth System employees), and uninsured. Smoking status was defined as current, former, or never/unknown.

\section{Outcomes}

We evaluated 4 outcomes: (1) emergency department visit likely related to COVID-19 subsequent to hotline telehealth visit, (2) hospitalization because of COVID-19 subsequent to hotline telehealth visit, (3) SARS-CoV-2 polymerase chain reaction (PCR) test ordered subsequent to telehealth visit, and (4) positive SARS-CoV-2 PCR test subsequent to telehealth visit. Emergency department visits and hospitalizations were collected from the electronic record of our care system and other care systems in our region using the same electronic record vendor (Epic) via a vendor-specific information exchange feature. Data included all emergency visits and hospitalizations March 13 to April 30. Analyses were not time limited (eg, by the number of days after a telehealth visit).

\section{Statistical Analyses}

Patient disposition following physician visits was reported descriptively in 3 categories: patient advised on COVID-19 isolation and home care, patient advised to visit the emergency department, or other (unlikely to have a viral respiratory illness or illness related to COVID-19). Visit disposition and patient characteristics were then incorporated as explanatory variables into a series of multivariable logistic regression models for each of the 4 outcomes described above. The models were used to calculate odds ratios and $95 \%$ confidence intervals to measure the association between each explanatory variable after adjusting for other model covariates and each outcome of interest. Regression analyses were conducted using SAS version 9.4 (Cary, NC). Missing data were handled with listwise deletion. All statistical tests are 2 tailed with significance defined as $P<.05$.

\section{Results}

The complete study flow and most common visit dispositions are represented in Figure 1 Between March 13 and April 20, 2020, there were a total of 12,512 calls to the $\mathrm{RN}$ triage line from 10,112 unique individuals. A total of $5027 \mathrm{RN}$ triage calls from 4213 patients were referred for a telehealth physician visit. An additional 96 physician telehealth visits had no preceding $\mathrm{RN}$ triage call. Demographic characteristics of the nurse triageonly and the physician COVID-19 line patients are presented in Table 1. Daily call volume was steady throughout the 5-week study period and the cumulative increase continued almost parallel to growth in confirmed COVID-19 cases for Cuyahoga County (Figure 2).

For all calls, common reasons for the call were cough (22.3\%), advice/health education (15.6\%), difficulty breathing $(6.1 \%)$, fever $(4.7 \%)$, and flulike illness (4.0\%). Similarly, the most common RN protocols used were for cough $(11.0 \%)$, chest pain (4.8), sore throat $(4.0 \%)$, abdominal pain $(3.4 \%)$, and fever $(3.3 \%)$. Among all calls to the $\mathrm{RN}$ triage line, $38 \%$ were referred for a same-day physician telehealth visit. Ten percent were advised to go immediately to the emergency department, and $52 \%$

Table 1. Descriptive Characteristics of CovID-19 Hotline Patients $(\mathrm{n}=10,208)$

\begin{tabular}{|c|c|c|c|}
\hline Characteristic & All & $\begin{array}{l}\text { RN Triage } \\
\text { Line only }\end{array}$ & $\begin{array}{c}\text { COVID-19 } \\
\text { MD Line }\end{array}$ \\
\hline Individuals & 10,208 & 5995 & 4213 \\
\hline Mean age, years & 41.9 & 41.9 & 42.0 \\
\hline Female, \% & 67.4 & 66.8 & 68.3 \\
\hline Smoking status, \% & 20.3 & 20.3 & 20.3 \\
\hline Current smoker & 28.0 & 29.3 & 26.1 \\
\hline Former smoker & 44.8 & 44.5 & 45.3 \\
\hline Never-smoker & 6.9 & 5.9 & 8.3 \\
\hline \multicolumn{4}{|c|}{ Unknown/not asked } \\
\hline Race, $\%$ & 51.2 & 47.8 & 56.0 \\
\hline White & 38.0 & 41.9 & 32.5 \\
\hline Black & 5.3 & 5.4 & 5.3 \\
\hline Hispanic & 5.5 & 4.9 & 6.2 \\
\hline \multicolumn{4}{|l|}{ Other/unknown } \\
\hline Insurance, \% & 37.0 & 31.7 & 44.1 \\
\hline Commercial & 16.7 & 19.5 & 12.9 \\
\hline Medicare & 35.2 & 39.3 & 29.6 \\
\hline Medicaid & 10.6 & 8.7 & 13.1 \\
\hline Uninsured other & 0.6 & 0.8 & 0.3 \\
\hline
\end{tabular}

Note: 96 patients had a COVID line call without a prior registered nurse triage call. 
Figure 2. CoVID-19 Cases (Hotline and Cuyahoga County) and Hospitalizations (County). CoVID line calls are derived from electronic health record data. Cuyahoga County case and hospitalization data were provided by the Cuyahoga County Board of Health and are available at https://www.ccbh.net/coronavirus/.

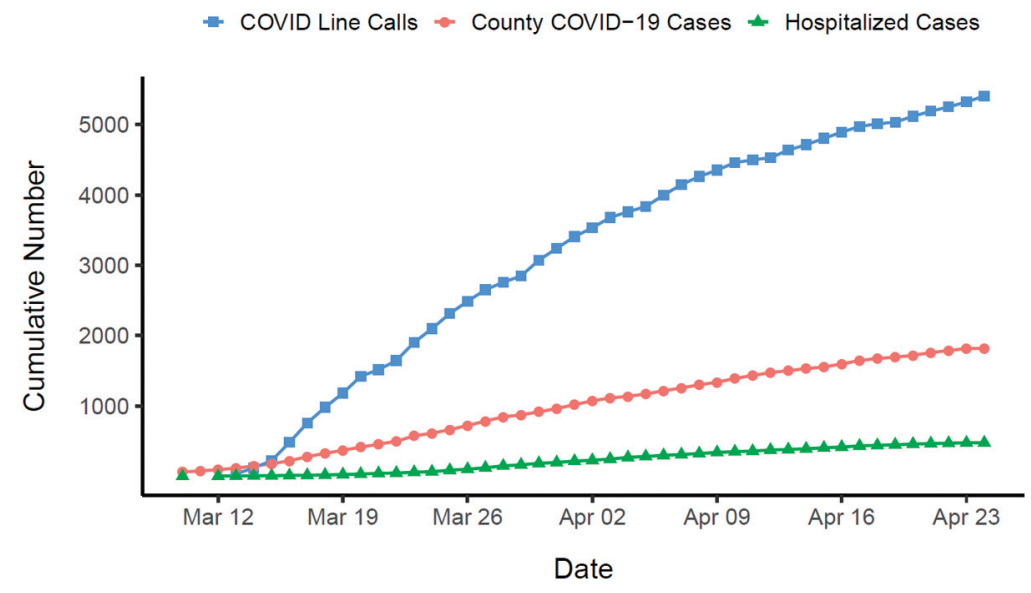

were not COVID-19 related or required no additional care. Of the hotline callers, $482(5 \%)$ had 1 or more COVID tests reported with 69 (14\%) testing positive. A total of 287 telehealth patients (7\%) had a subsequent emergency department visit, and 44 (1\%) were hospitalized with a COVID-19 diagnosis. Among those patients advised to stay at home at their first physician hotline visit, $83 \%$ recorded no further face-to-face clinical encounters.

For the patients with a physician phone visit, common visit diagnoses included respiratory symptoms (43.0\%), Upper Respiratory Infection (URI) unspecified type (5.7\%), cough (5.5\%), viral URI with cough (3.6\%), and sore throat $(2.1 \%)$. Less than $2 \%$ of scheduled phone calls were no show. Among all physician telephone visits, $79 \%$ were advised to stay at home, self-isolate, and continue monitoring symptoms. In $14 \%$ of visits, physicians felt the patient was unlikely to have a COVID-related problem, and 5\% were advised to go to the emergency department or seek additional nonemergency care or testing.

Of the 4213 patients with a physician phone visit, $3713(88 \%)$ received 1 or more care-coordinator follow-up calls. At follow-up, 92\% of patients reported their symptoms had not worsened and $67 \%$ reported having connected with the follow-up recommended on the initial physician phone visit. Some patients (16\%) reported feeling overwhelmed, anxious, or isolated because of COVID19, and 111 patients (3\%) reported needing help to manage their basic needs. Those expressing interest in receiving help for those needs were offered services from the MetroHealth Institute for H.O.P.E., which provided patients with multiple services including food deliveries ( $\mathrm{n}=92)$, behavioral health telephone visits $(n=49)$, and faithbased comfort calls from pastoral care personnel $(\mathrm{n}=37)$. Prescription deliveries were available for all patients and clinical social work support was available as needed.

Findings from multivariable logistic regression models including patient demographics, smoking status, and physician telephone disposition are presented in Table 2. Emergency department visits and hospitalizations were positively associated with a telehealth visit disposition, indicating that the patient was high risk and should seek emergency care. Receipt of COVID-19 testing was associated with older age, current smoking status, employee health insurance, and a telephone visit disposition of high risk or self-isolation. Finally, among those who received testing, a positive test result was associated with older age.

\section{Discussion}

In this report, we demonstrate the feasibility and effectiveness of a large-scale, physician-staffed hotline in providing care and disseminating information to the general public of a large metropolitan area amid a global pandemic. Strengths of our study include a large sample consisting of many individuals who were not tested despite concerns about having COVID-19, care process data from standardized 
Table 2. Logistic Regression Results for Patient Characteristics Associated with (1), Emergency Department Visit (2), Coronavirus 2019 Hospitalization (3), Severe Acute Respiratory Syndrome Coronavirus Polymerase Chain Reaction Testing, and (4) Positive Severe Acute Respiratory Syndrome Coronavirus Polymerase Chain Reaction Test

\begin{tabular}{|c|c|c|c|c|c|c|c|c|c|c|c|c|}
\hline \multirow{2}{*}{$\begin{array}{l}\text { Variables } \\
\text { Parameter }\end{array}$} & \multicolumn{3}{|c|}{$\begin{array}{c}\text { ED Visit } \\
\mathrm{N}=2569, \mathrm{c}=0.65\end{array}$} & \multicolumn{3}{|c|}{$\begin{array}{c}\text { Hospitalization } \\
\mathrm{N}=2569, \mathrm{c}=0.77\end{array}$} & \multicolumn{3}{|c|}{$\begin{array}{c}\text { SARS-CoV-2 Test } \\
\mathrm{n}=2569, \mathrm{c}=0.76\end{array}$} & \multicolumn{3}{|c|}{$\begin{array}{c}\text { Positive SARS-CoV-2 } \\
\mathrm{N}=249, \mathrm{c}=0.67 \\
\end{array}$} \\
\hline & $\begin{array}{l}\text { Odds } \\
\text { Ratio }\end{array}$ & $95 \% \mathrm{CI}$ & $P$ & $\begin{array}{l}\text { Odds } \\
\text { Ratio }\end{array}$ & $95 \% \mathrm{CI}$ & $P$ & $\begin{array}{l}\text { Odds } \\
\text { Ratio }\end{array}$ & $95 \% \mathrm{CI}$ & $P$ & $\begin{array}{l}\text { Odds } \\
\text { Ratio }\end{array}$ & $95 \% \mathrm{CI}$ & $P$ \\
\hline Age (per 10 years) & 1.14 & $0.95-1.37$ & .15 & 1.09 & $0.77-1.54$ & .62 & 1.13 & $1.03-1.24$ & .01 & 1.47 & $1.10-1.96$ & .01 \\
\hline \multicolumn{13}{|l|}{ Sex (female) } \\
\hline Male & 1.29 & $0.72-2.22$ & .42 & 0.70 & $0.22-2.25$ & .55 & 1.20 & $0.88-1.62$ & .24 & 1.40 & $0.68-2.93$ & .36 \\
\hline \multicolumn{13}{|l|}{ Race } \\
\hline \multicolumn{13}{|l|}{ NH white } \\
\hline NH black & 1.03 & $0.56-1.87$ & .93 & 0.85 & $0.28-2.60$ & .77 & 0.84 & $0.61-1.15$ & .28 & 1.68 & $0.78-3.62$ & .19 \\
\hline Hispanic & 1.57 & $0.59-4.19$ & .36 & 1.19 & $0.14-9.80$ & .87 & 0.61 & $0.30-1.27$ & .19 & 0.68 & $0.08-6.01$ & .73 \\
\hline Other & 0.93 & $0.28-3.14$ & .90 & 1.17 & $0.14-9.67$ & .88 & 1.28 & $0.75-2.17$ & .36 & 0.66 & $0.16-2.66$ & .56 \\
\hline \multicolumn{13}{|l|}{$\begin{array}{l}\text { Insurance type } \\
\text { (commercial) }\end{array}$} \\
\hline Medicaid & 0.86 & $0.38-1.95$ & .72 & 2.71 & $0.27-27.24$ & .40 & 0.97 & $0.60-1.56$ & .90 & 2.62 & $0.79-8.74$ & .12 \\
\hline Medicare & 1.18 & $0.47-3.00$ & .72 & 4.98 & 0.48 to 51.63 & .18 & 1.11 & $0.63-1.94$ & .72 & 0.56 & $0.12-2.61$ & .46 \\
\hline Employee & 1.55 & $0.69-3.46$ & .29 & 11.45 & 1.35 to 96.95 & .02 & 7.13 & $4.82-10.54$ & $<.01$ & 1.35 & $0.49-3.75$ & .56 \\
\hline Uninsured & 1.76 & $0.74-4.22$ & .20 & 4.26 & 0.38 to 47.79 & .23 & 1.15 & $0.68-1.96$ & .60 & 1.55 & $0.38-6.34$ & .54 \\
\hline \multicolumn{13}{|l|}{$\begin{array}{l}\text { COVID-19 line } \\
\text { disposition (other) }\end{array}$} \\
\hline Stay at home & 1.95 & $0.91-4.21$ & .09 & 0.77 & $0.23-2.54$ & .66 & 1.90 & $1.29-2.78$ & $<.01$ & 1.08 & $0.40-2.87$ & .89 \\
\hline High risk & 4.73 & $1.37-16.39$ & .01 & 7.77 & $1.61-37.50$ & .01 & 8.78 & $4.39-17.53$ & $<.01$ & 0.48 & $0.07-3.14$ & .44 \\
\hline \multicolumn{13}{|l|}{$\begin{array}{l}\text { Smoking (never, } \\
\text { unknown) }\end{array}$} \\
\hline Current & 0.67 & $0.32-1.40$ & .29 & 0.76 & $0.15-3.78$ & .73 & 0.41 & $0.26-0.65$ & $<.01$ & 0.53 & $0.14-2.03$ & .36 \\
\hline Former & 0.70 & $0.36-1.36$ & .29 & 1.46 & $0.47-4.56$ & .52 & 0.65 & $0.46-0.92$ & .02 & 0.70 & $0.29-1.69$ & .42 \\
\hline
\end{tabular}

ED, emergency department; SARS-CoV-2, severe acute respiratory syndrome coronavirus; NH, non-Hispanic; COVID-19, coronavirus 2019; CI, confidence interval.

protocols, and outcome data collected from the electronic health records from multiple care systems.

Recent reports have focused on use of a selfadministered survey as a mechanism for conserving personal protective equipment, citing the difficulty of rapidly creating robust telehealth infrastructure. ${ }^{20}$ In contrast, our results indicate that robust, comprehensive, and hospital-integrated telehealth are an effective form of health services during the acute phase of a pandemic and beyond. Telephone hotlines require a minimum of technological capabilities, can be implemented rapidly, and are accessible to those who may not have internet access, especially when facilities with public internet access such as libraries are closed. Past research has indicated that telephone calls yield similar patient health outcomes when compared with video-based appointments. ${ }^{21}$
Although some communities have described higher rates of infection, hospitalization, and death among low-income and racial and ethnic minority patients, data from our hospital system in northeast Ohio do not indicate any observed racial or socioeconomic disparities in care process or outcome. Our results indicate that employees of the health care system who called the hotline were more likely to be tested, but this is a function of system policies, which referred employees with suspected or proven COVID-19 exposure to call the hotline as well as the need to protect patients from potentially infected care personnel. In our population, we found no association between current smoking status and emergency department visits or hospitalization after a telephone encounter for patients with COVID-19-related symptoms. This finding is 
consistent with a review of several prior studies outside the United States that found little evidence for the influence of tobacco smoking on COVID-19 outcomes. $^{22}$ However, prior investigations and reviews have been confined to case series designs derived from data reported to public health agencies or to hospitalized patients, which limit the ability to include important covariates and exclude untested patients reporting acute respiratory illness. $^{22-25}$

The results of our study have several important limitations. We report on a hotline from a single, large health care system serving an urban area of northeast Ohio. Our findings may not be generalizable to other health systems or areas because of variation in COVID-19 government and public health response. Although we included data available through Epic's Care Everywhere health information exchange, we may not have captured all data from other regional care systems, and some patients may have received services at other hospitals or emergency departments that are not available for our analyses. However, 2 of the 3 major health systems in Cuyahoga County use the Epic electronic health record, and COVID-19 test results from all 3 systems (covering more than $90 \%$ of all acute care) were captured in our data, suggesting any missed utilization may be minimal. Our study data did not include sufficient call log information to examine wait times and dropped calls, so it is possible that at times of peak volume some callers were unable to get through on the first attempt. However, this is mitigated in part because of the fact that telehealth visits with physicians were scheduled visits rather than telephone drop-in.

Prior research has demonstrated that telephone hotlines are a convenient and efficient mode of information distribution from health care providers to a large population, and our data indicate that a significant number of our study population sought advice or information as the primary reason for calling. ${ }^{8}$ Readily accessible information and medical care during a pandemic is a crucial public health function because it can directly reduce demand for emergency services and efficiently provide large numbers of symptomatic and potentially infected persons guidance about how to stay at home and protect themselves and others. The vast majority of patients with telehealth visits in our population were advised to seek home care, avoiding the need to seek care at a walk-in clinic or the emergency department.
We did not find evidence of disparities by race and ethnicity or insurance type, which leads us to conclude that telephone hotline services are an accessible and equitable form of care delivery that reaches lower socioeconomic status communities during the COVID-19 pandemic. Services that depend on internet access, such as patient portals, have been shown to have lower usage among older adults and racial and ethnic minorities, the very populations most affected by COVID-19 severe disease in the United States. ${ }^{26,27}$ Furthermore, results of our multivariable models indicate that primary care physicians assessing patients over the phone make effective decisions about which patients will require use of emergency services and which can be safely managed at home. By transforming a large number of potential in-person visits to telephone visits, our study demonstrates that it is possible to conserve increasingly scarce personal protective equipment, appropriately limit testing, and potentially prevent the further spread of infection to patients and health care workers in otherwise busy in-person care settings. DeVoe et $\mathrm{al}^{28}$ recently published a plea for a regional telehealth primary care extension infrastructure to address the demands being placed on care systems. Our findings provide evidence for the effectiveness of such an approach and suggest that policy makers and medical and public health leaders should consider widespread implementation of physician-staffed telehealth services as a key component of effective, equitable pandemic response.

Authors would like to acknowledge the contributions of Sandra Andrukat, Kim Bauchens, RN, MSN, Shari Bolen MD, Karen Cook, Nick Dreher MD, and Ryan Johnson.

To see this article online, please go to: http://jabfm.org/content/ 34/Supplement/S95.full.

\section{References}

1. Riou J, Althaus CL. Pattern of early human-tohuman transmission of Wuhan 2019 novel coronavirus (2019-nCoV). Euro Surveill 2020;25(4):2000058. December 2019 to January 2020 [published correction appears in Euro Surveill. 2020 Feb;25(7)].

2. Johns Hopkins University Coronavirus Resource Center. COVID-19 global map. https://coronavirus. jhu.edu/map.html.

3. Yang P, Wang X. COVID-19: a new challenge for human beings. Cell Mol Immunol 2020;17:555-63.

4. Eppes CS, Garcia PM, Grobman WA. Telephone triage of influenza-like illness during pandemic 
$2009 \mathrm{H} 1 \mathrm{~N} 1$ in an obstetric population. Am J Obstet Gynecol 2012;207:3-8.

5. Spaulding AB, Radi D, Macleod H, et al. Design and implementation of a statewide influenza nurse triage line in response to pandemic $\mathrm{H} 1 \mathrm{~N} 1$ influenza. Public Health Rep 2012;127:532-40.

6. Spaulding AB, Radi D, Macleod $\mathrm{H}$, et al. Satisfaction and public health cost of a statewide influenza nurse triage line in response to pandemic H1N1 influenza. PLoS One 2013;8:e50492.

7. Koonin LM, Hanfling D. Broadening access to medical care during a severe influenza pandemic: the CDC Nurse Triage Line Project. Biosecur Bioterror 2013;11:75-80.

8. Lin L, Jung M, McCloud RF, Viswanath K. Media use and communication inequalities in a public health emergency: a case study of 2009-2010 pandemic influenza a virus subtype H1N1. Public Health Rep 2014;129:49-60.

9. Whaibeh E, Mahmoud H, Naal H. Telemental health in the context of a pandemic: the COVID-19 experience. Curr Treat Options Psych 2020;7:19895.[published online ahead of print, $2020 \mathrm{Apr} 2$ ].

10. Zhang D, Wang G, Zhu W, et al. Expansion of telestroke services improves quality of care provided in super rural areas. Health Aff (Millwood) 2018;37:200513. Dec.

11. Lurie N, Carr BG. The role of telehealth in the medical response to disasters. JAMA Intern Med 2018;178:745-6. Jun 1.

12. Ohannessian R. Telehealth: potential applications in epidemic situations. Eur Res Telehealth/La Recherche Européenne En Télémédecine 2015;4:95-8.

13. Rockwell KL, Gilroy AS. Incorporating telehealth as part of COVID-19 outbreak response systems. Am J Managed Care 2020;26:147-8. Apr 1.

14. Turer RW, Jones I, Rosenbloom ST, Slovis C, Ward MJ. Electronic personal protective equipment: a strategy to protect emergency department providers in the age of COVID-19. JAMIA 2020;27:967-71.

15. Moazzami B, Razavi-Khorasani N, Moghadam AD, Farokhi E, Rezaei N. COVID-19 and telehealth: immediate action required for maintaining healthcare providers well-being. J Clin Virol 2020;126:104345.

16. Hollander JE, Carr BG. Virtually perfect? Telehealth for Covid-19. N Engl J Med 2020;18:1679-81.

17. Judson TJ, et al. Rapid design and implementation of an integrated patient self-triage and self-scheduling tool for COVID-19. JAMIA 2020.

18. Reeves JJ, et al. Rapid response to COVID-19: health informatics support for outbreak management in an academic health system. JAMIA 2020.
19. Erickson D, Reid C, Nelson L, O'Shaughnessy A, Berube A. The enduring challenge of concentrated poverty in America: case studies from communities across the US. Washington (DC): Federal Reserve System; 2008.

20. Perez-Alba E, Nuzzolo-Shihadeh L, EspinosaMora JE, Camacho-Ortiz A. Use of self-administered surveys through QR code and same center telehealth in a walk-in clinic in the era of COVID-19. Oxford (England): OUP Academic, Oxford University Press. http://dx.doi.org/academic.oup.com/jamia/article/ doi/10.1093/jamia/ocaa054/5819556.

21. Rush KL, Howlett L, Munro A, Burton L. Videoconference compared to telephone in healthcare delivery: a systematic review. Int $\mathrm{J}$ Med Inform 2018;118:44-53. Oct. Epub 2018 Jul 25.

22. Emami A, Javanmardi F, Pirbonyeh N, Akbari A. Prevalence of underlying diseases in hospitalized patients with COVID-19: a systematic review and meta-analysis. Arch Acad Emerg Med 2020;8(1): e35.

23. Richardson S, Hirsch JS, Narasimhan M, Northwell COVID-19 Research Consortium. Presenting characteristics, comorbidities, and outcomes among 5700 patients hospitalized with COVID-19 in the New York City area. JAMA. Published online 2020; 323:2052-2059.

24. Myers LC, Parodi SM, Escobar GJ, Liu VX. Characteristics of hospitalized adults with COVID-19 in an Integrated health care system in California. JAMA. Published online 2020; $323: 2195$.

25. Centers for Disease Control and Prevention. Severe outcomes among patients with coronavirus disease 2019 (COVID-19)_United States, February 12-March 16, 2020. Morb Mort Wkly Rep. Updated March 27, 2020. Accessed March 27, 2020. https://www.cdc.gov/mmwr/volumes/ 69/wr/mm6912e2.htm.

26. Perzynski AT, Roach MJ, Shick S, et al. Patient portals and broadband internet inequality. JAMIA 2017;24:927-32. Sep 1.

27. Rodriguez JA, Lipsitz SR, Lyles CR, Samal L. Association between patient portal use and broadband access: a national evaluation. JGIM 2020;10:1-2. Jan.

28. DeVoe JE, Cheng A, Krist A. Regional strategies for academic health centers to support primary care during the COVID-19 pandemic: a plea from the front lines. JAMA Health Forum 2020. Accessed April 28, 2020;1:e200423. April 8. https://jamanetwork.com/ channels/health-forum/fullarticle/2764405. 